\title{
Poor ability to resist tempting calorie rich food is linked to altered balance between neural systems involved in urge and self-control
}

\author{
Qinghua He $e^{1,2}$, Lin Xiao ${ }^{2}$, Gui Xue ${ }^{3}$, Savio Wong ${ }^{4}$, Susan L Ames ${ }^{5}$, Susan M Schembre ${ }^{6}$ and Antoine Bechara ${ }^{*}$
}

\begin{abstract}
Background: The loss of self-control or inability to resist tempting/rewarding foods, and the development of less healthful eating habits may be explained by three key neural systems: (1) a hyper-functioning striatum system driven by external rewarding cues; (2) a hypo-functioning decision-making and impulse control system; and (3) an altered insula system involved in the translation of homeostatic and interoceptive signals into self-awareness and what may be subjectively experienced as a feeling.
\end{abstract}

Methods: The present study examined the activity within two of these neural systems when subjects were exposed to images of high-calorie versus low-calorie foods using functional magnetic resonance imaging (fMRI), and related this activity to dietary intake, assessed by 24-hour recall. Thirty youth (mean BMl $=23.1 \mathrm{~kg} / \mathrm{m}^{2}$, range $=19.1-33.7$; age $=19.7$ years, range $=14$ - 22) were scanned using fMRI while performing food-specific go/nogo tasks.

Results: Behaviorally, participants more readily pressed a response button when go trials consisted of high-calorie food cues (HGo task) and less readily pressed the response button when go trials consisted of low-calorie food cues (LGo task). This habitual response to high-calorie food cues was greater for individuals with higher BMI and individuals who reportedly consume more high-calorie foods. Response inhibition to the high-calorie food cues was most difficult for individuals with a higher BMI and individuals who reportedly consume more high-calorie foods. fMRI results confirmed our hypotheses that (1) the "habitual" system (right striatum) was more activated in response to high-calorie food cues during the go trials than low-calorie food go trials, and its activity correlated with participants' BMl, as well as their consumption of high-calorie foods; (2) the prefrontal system was more active in nogo trials than go trials, and this activity was inversely correlated with BMl and high-calorie food consumption.

Conclusions: Using a cross-sectional design, our findings help increase understanding of the neural basis of one's loss of ability to self-control when faced with tempting food cues. Though the design does not permit inferences regarding whether the inhibitory control deficits and hyper-responsivity of reward regions are individual vulnerability factors for overeating, or the results of habitual overeating.

Keywords: Decision making, Obesity, fMRI, Food, Habitual system, Prefrontal system

\section{Background}

Overweight and obesity, expressed as above-normal body mass index $\left(\mathrm{BMI}>24.9 \mathrm{~kg} / \mathrm{m}^{2}\right)$, is a public health challenge worldwide. In the United States, nearly $70 \%$ of adults are overweight or obese [1]. Overweight and obesity are associated with increased risk for cardiovascular/metabolic

\footnotetext{
*Correspondence: bechara@usc.edu

${ }^{2}$ Brain and Creativity Institute and Department of Psychology, University of Southern California, Los Angeles, CA, USA

Full list of author information is available at the end of the article
}

diseases, as well as some cancers [2]. Although there is no clear explanation of the primary cause of overweight and obesity, excessive weight gain is the known result of chronic positive energy imbalances favoring calories consumed over calories expended [3]. Thus, it is important to identify underlying mechanisms that relate to behaviors associated with excessive energy intake in order to address the obesity epidemic. Much research to date has explored the underlying influences of genetic, hormonal, and metabolic mechanisms related to 
obesity and dietary intake [4-9]. Fewer studies have sought to understand the underlying, and potentially modifiable, neural mechanisms that motivate decisions about "what" and "how much" to eat [10-19].

Mounting evidence suggests that the difficulty to resist highly palatable, calorie-rich foods represents a special case of addictive behavior with similarities to other addictions [20-24]. Several studies have shown that individuals who eat excessively are unable to make optimal food-related choices [25,26], characterized by a tendency to choose the immediate reward of a food item at the expense of potentially long-term negative consequences [27]. Based on these findings, we hypothesized that a loss of self-control or inability to resist tempting/rewarding foods, and the development of less healthful eating habits (e.g., greater intake of high-calorie foods), may be explained by three key neural systems: (1) a hyperfunctioning striatum driven by external rewarding cues, including highly rewarding foods. We have referred to this neural system as the "impulsive" system [28-31]. This is the same system that has been shown, in animal models, to be responsible for the development of automatic and habitual behaviors in response to reward cues [32,33]; (2) a hypo-functioning decision-making and impulse control system, which includes the mesial orbitofrontal cortex, the sub-genual regions of the anterior cingulate cortex (ACC), and adjacent areas within the ventral medial prefrontal cortex. In addition, inhibition control and response inhibition have also been linked to lateral regions of the prefrontal cortex [34-39]. We have referred to this neural system as the "reflective" system or prefrontal system [28]; and (3) based on more recent evidence on the effects of brain lesions on smoking behavior [40], we hypothesized that the strength of the two previously outlined neural systems can be modulated by the insula system involved in urge and craving, which includes the anterior insula. Indeed, the anterior insula is thought to be involved in the translation of homeostatic and interoceptive signals into self-awareness of subjective feelings [41-45]. Accordingly, we have proposed that interoceptive signals triggered by food deprivation, or by exposure to food cues, are relayed to the insular cortex and translated into craving and what may become subjectively experienced as an intense urge to eat. Consistent with this conceptualization, neuroimaging studies have shown the impulsive system (striatum) to be consistently more active during exposure to high-calorie foods when compared to low-calorie foods or control images [13,16,46-55]. This effect is greater for overweight versus normal weight participants [47,53,56,57], and could potentially predict short-and long-term outcomes in weight-loss programs [58]. On the other hand, an increasing number of studies suggest that activity within the prefrontal system is also altered in response to food cues $[59,60]$. These results are consistent with the idea that these systems, which were previously shown to have altered activity in cases of other substance addictions, also show some altered responses to visual food cues in obese individuals [46,61-65].

The present study used functional magnetic resonance imaging (fMRI) techniques to investigate brain activity related to the neural systems described above during food-specific go/nogo tasks consisting of high- and lowcalorie food cues. Specifically, we tested hypotheses related to the activities of the impulsive (striatum) and reflective (prefrontal) neural systems in response to images of high-calorie versus low-calorie food cues, and related these activities to dietary intake. Participants were adolescents and young adults who represent an intriguing group to study given the relatively delayed maturation of the prefrontal cortex [66-68], and, as a result, the potential for making disadvantageous food choices. Indeed, this population has a tendency to make less healthful food choices [69], and such choices are facilitated by school environments and university campuses where accessibility to highly palatable, high-calorie food options is relatively unrestricted.

\section{Methods}

\section{Participants}

Thirty (17 female) healthy adolescents and young adults aged 19.7 years $(S D=1.7$, range $=14-22$ ) years were recruited (see Table 1). Their average BMI was $23.1 \mathrm{~kg} / \mathrm{m}^{2}$ $(\mathrm{SD}=3.0$, range $=19.1-33.7)$. None of the participants were currently receiving clinical treatment for obesity. All participants had normal or corrected-to-normal vision. To rule out participants with certain neuropsychiatric disorders, medications, or health issues that could impact the neuroimaging results, we used (1) the Structured Clinical Interview for DSM-IV (SCID) to exclude individuals who meet the criteria for current psychoses, anxiety, or bipolar disorders, as well as the criteria for substance abuse; and (2) a 41-item questionnaire that asks for the presence of diabetes, hypertension, lungs, heart, kidney, or liver disease. The same questionnaire

Table 1 Descriptive statistics of all behavior measures and dietary intake

\begin{tabular}{lcccc}
\hline & Mean & SD & Range & Gender difference \\
\hline BMI $\left(\mathbf{k g} / \mathbf{m}^{\mathbf{2}}\right)$ & 23.1 & 3.0 & $19.1-33.7$ & $\mathrm{t}=1.67, \mathrm{p}=.11$ \\
IQ & 117.5 & 9.5 & $103-136$ & $\mathrm{t}=.85, \mathrm{p}=.40$ \\
SOPT & 65.1 & 3.5 & $56-70$ & $\mathrm{t}=.31, \mathrm{p}=.76$ \\
Hungry Rating & 2.6 & 2.0 & $1-4$ & $\mathrm{t}=.86, \mathrm{p}=.40$ \\
NDSR Low-Calorie Foods & 2.4 & 1.6 & $.1-7.5$ & $\mathrm{t}=2.76, \mathrm{p}<.01^{* *}$ \\
NDSR High-Calorie Foods & 1.8 & 1.3 & $0-5.7$ & $\mathrm{t}=.64, \mathrm{p}=.53$ \\
\hline
\end{tabular}

** $\mathrm{p}<.01$. SD Standard Deviation, BMI Body mass index, SOPT Self-ordered pointing task, NDSR Nutrition data system for research. 
also asks for a history of head trauma, or other neurological disease, and also records the use of current medications, smoking (nicotine), alcohol, and caffeine, and whether they are currently receiving clinical treatment for obesity or following a certain diet. Subjects who meet a psychiatric diagnosis, or report a history of head injury or neurological disease, or current use of medications that impact the central nervous system, including nicotine, or are currently in clinical treatment for obesity and follow a certain diet are excluded. All participants and their parents (for participants under 18) gave informed consent to the study procedures, which were approved by the University of Southern California Institutional Review Board (reference number UP-10-00052).

\section{Procedures}

Participants were asked to come to the lab for two sessions (Figure 1A). During the first visit, participants (and a parent for those under 18) were asked to complete and sign the consent form(s), and complete the SCID and behavioral tasks. Participants were then scheduled to return for the fMRI scan session. Twenty-six subjects completed their scan in the morning between the hour of $10 \mathrm{am}$ and $11 \mathrm{am}$, and only 4 subjects were scanned in the afternoon due to scheduling restraints. Participants were asked to refrain from any intense physical activity prior to scanning for 24 hours. Participants were asked to eat normally and to have their usual meal before they arrived for the fMRI session. Prior to the fMRI scan, height and weight were assessed using standard procedures, a 24-hour dietary recall was conducted, and participants rated their hunger level on a scale ranging from 1 (not hungry at all) to 10 (very hungry) to ensure that the participant was not in a deprived state. Subjects who provided a hunger rating $>5$ were asked to reschedule their scan and to return after they had first consumed a normal meal. Thus all scanned participants rated their hunger as $\leq 4$, with a mean score of 2.6.

\section{Measures}

\section{Behavior tasks}

Participants were asked to complete two behavioral tasks (see Table 1). The Wechsler Abbreviated Scale of Intelligence WASI, [70] was used as an estimate for IQ, and the SelfOrdered Pointing Task SOPT, [71] was used as an index of working memory and executive functioning.

\section{Dietary intake}

A single, in-person 24-hour dietary recall was conducted by trained research staff using a multipass method facilitated by the Nutrition Data System for Research software NDSR, [72,73]. All of the recalls were reported for a weekday. The software includes a dietary supplement assessment module so that nutrient intake from both food and supplemental sources may be captured and quantified. Based on the NDSR nutrient totals report, no

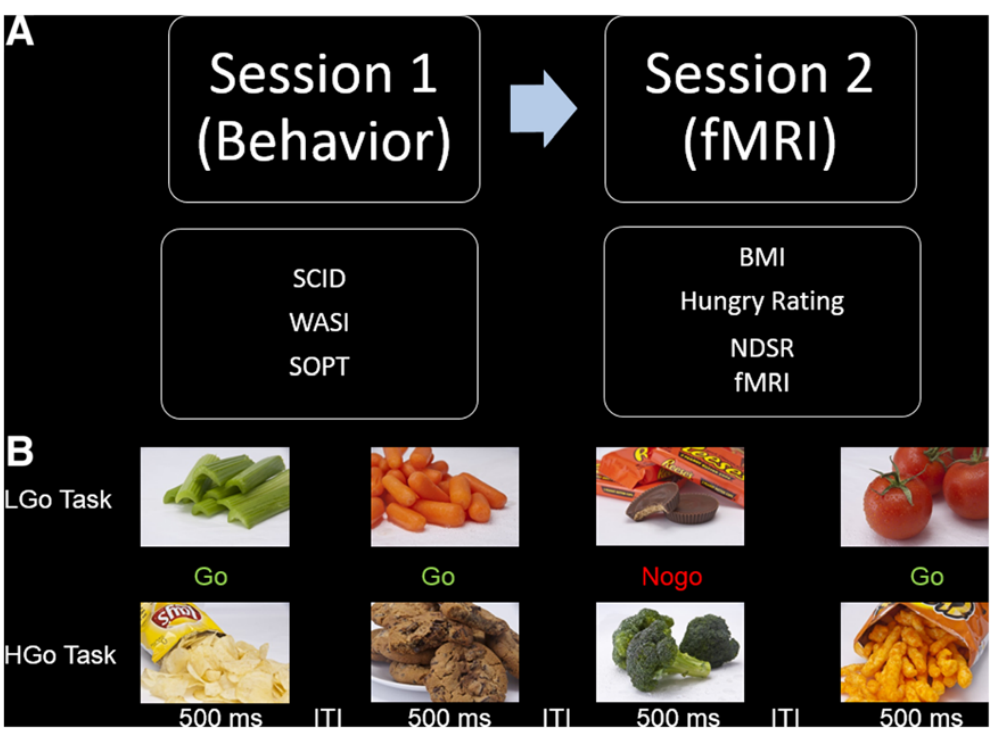

Figure 1 Design of the study. A) The schematic of the procedure. Participants were asked to visit the lab for two sessions: one behavior session and one fMRI session. B) The illustration of the event-related food-specific go/nogo tasks 1) low-calorie food go/high-calorie food nogo task (LGo task), and 2) high-calorie food go/low-calorie food nogo task (HGo task). Participants were asked to press a button as soon as possible to the go trials (vegetable pictures in LGo task and snack pictures in HGo task) and withhold the response to the nogo trials (snack pictures in LGo task and vegetable pictures in HGo task). The order of tasks was counterbalanced across subjects. SCID: structured clinical interview for DSM-IV; WASI: Wechsler abbreviated scale of intelligence; SOPT: self-ordered pointing task; BMI: body mass index; NDSR: nutrition data system for research; fMRI: functional magnetic resonance imaging; ITI: intertribal interval. 
individual was identified as reporting an implausible total energy intake $(<500$ or $>7000 \mathrm{kcal})$ as suggested by a previous study [74]. Total daily servings of low-calorie foods and high-calorie foods were computed by summing total intake of fruits and vegetables (servings/day) and total intake of fatty foods and sugar-sweetened foods (servings/day), respectively. For the analyses, low and high-calorie food consumption (servings/day) were calorieadjusted for total energy intake, as estimated by the dietary recall, and are reported as servings per $1000 \mathrm{kcals}$ (servings $/ 1000 \mathrm{kcals}$ ). The calorie-adjustment was performed to ensure that higher levels of intake were not artifacts of higher energy expenditure, as related to age or high levels of activity.

\section{fMRI tasks}

Participants performed two food-specific go/nogo tasks in the scanner as follows: 1) a low-calorie food go and high-calorie food nogo task (LGo task), and 2) highcalorie food go and low-calorie food nogo task (HGo task). This go/nogo paradigm allows examination of the inhibition of prepotent responses to appetizing food items. Participants were asked to press a button as soon as possible to the go trials (low-calorie food pictures in LGo task and high-calorie food pictures in HGo task), and to withhold responses to the nogo trials (high-calorie food pictures in LGo/HNogo task and low-calorie food pictures in HGo/LNogo task). Examples of lowcalorie food images included cucumbers, celery, broccoli, and carrots. Examples of high-calorie food images included chocolate bars, cookies, ice cream, and potato chips. All images of the foods observed are commonly available in everyday life (Figure 1B).

Each task consisted of 120 go trials (75\%) and 40 nogo trials (25\%). Nogo trials were presented in pseudo-randomized order, designed so that Nogo trials appeared with equal probability after $1-5$ consecutive Go trial presentations, and no two Nogo trials appeared consecutively. Each stimulus was presented for $500 \mathrm{~ms}$, followed by a fixation cross for 1.5 - 4 seconds with a mean of $2.5 \mathrm{~s}$. The sequence was optimized for design efficiency using an in-house program. Each task ran for 8 minutes. The order of two versions of go-nogo tasks was counterbalanced across subjects.

Following signal detection theory, the hit rate, false alarm rate, sensitivity index d' $\left(d^{\prime}=Z_{\text {hits rate }}-Z_{\text {false alarm rate }}\right)$ and decision bias $C\left[C=-0.5 \times\left(Z_{\text {hits rate }}+Z_{\text {false alarm rate }}\right)\right]$ were calculated for each task. The mean reaction time for go trials and nogo trials (false alarm trials only) for each task were also calculated. The reaction time for go trials served as an index for habitual responding to the stimuli, with longer reaction times indicating less habitual response; while decision bias $C$ served as an index of response inhibition, with higher values indicating better inhibitory control.

\section{fMRI protocol}

Prior to the scanning procedure, participants reviewed all stimuli used in the tasks and were informed by a research assistant of the category to which each stimulus belonged. During the fMRI scan, participants laid in the supine position on the scanner bed to view the task back-projected onto a screen through a mirror attached to the head coil. Foam pads were used to minimize head motion. Stimulus presentation and timing of all stimuli and response events were achieved using Matlab (Mathworks) and Psychtoolbox (www.psychtoolbox.org) on an IBM-compatible PC. Participants' responses were collected online using an MRI-compatible button box.

fMRI imaging was conducted in a 3 T Siemens MAGNETOM Tim/Trio scanner in the Dana and David Dornsife Cognitive Neuroscience Imaging Center at the University of Southern California. Blood oxygen level dependent (BOLD) functional scanning used a z-shim gradient echo EPI (echo planer imaging) sequence with PACE (prospective acquisition correction). This specific sequence is dedicated to reduce signal loss in the prefrontal and orbitofrontal areas. The PACE option can help reduce the impact of head motion during data acquisition. The parameters are: TR/TE $=2000 / 25 \mathrm{~ms}$; flip angle $=90^{\circ} ; 64 \times 64$ matrix size with resolution $3 \times$ $3 \mathrm{~mm}^{2}$. Thirty-one $3.5-\mathrm{mm}$ axial slices were used to cover the whole cerebral cortex and most of the cerebellum with no gap. The slices were tilted about $30^{\circ}$ clockwise along the AC-PC plane to obtain better signals in the orbitofrontal cortex. The anatomical T1-weighted structural scan was done $\left(\mathrm{TR} / \mathrm{TE}=1950 / 2.26 \mathrm{~ms}\right.$; flip angle $7^{\circ}$; 176 sagittal slices; spatial resolution $=1 \times 1 \times 1.95 \mathrm{~mm}$ ) for registration purpose.

\section{fMRI analysis}

Image preprocessing and statistical analysis were carried out using FSL package (www.fmrib.ox.ac.uk/fsl). fMRI images were realigned to compensate for small residual head movements that were not captured by the PACE sequence [75]. Translational movement parameters never exceeded 1 voxel in any direction for any participant. Data were spatially smoothed using a 5-mm full-width-halfmaximum (FWHM) Gaussian kernel. The data were filtered using a nonlinear high pass filter with a 100-second cut-off.

A two-step registration procedure was used whereby EPI images were first registered to the MPRAGE structural image, and then into standard MNI space, using affine transformations [75]. Registration from MPRAGE structural image to standard space was further refined using FNIRT nonlinear registration [76,77]. Statistical analyses were performed in the native image space, with the statistical maps normalized to the standard space prior to higher-level analyses. The data were modeled at 
the first level using a general linear model (GLM) within FSL's FILM module. Brain activation in every trial was modeled for go and nogo trials respectively in the single subject level. Error-related trials (misses and false alarms) were modeled together as a nuisance variable. The event onsets were convolved with the canonical hemodynamic response function (HRF, double-gamma) to generate regressors used in the GLM. Temporal derivatives were included as covariates of no interest to improve statistical sensitivity. Null events were not explicitly modeled, and therefore constituted an implicit baseline. The six movement parameters were also included as covariates in the model.

Higher-level analyses created cross-run contrasts for each subject using a fixed effects model. A 2 Task (Go vs Nogo) $\times 2$ Stimuli (Low-calorie vs High-calorie food cues) within-subject factor design was used. Higher level random-effects models were tested for group analyses using FMRIB's Local Analysis of Mixed Effect stage 1 only $[78,79]$ with automatic outlier detection [80]. First, a full $2 \times 2$ factor analysis was conducted at the group level to test for the main effects of task and stimuli as well as their interaction. Then, of particular interest to us, two additional hypotheses were evaluated at the group level as follows: (1) that high-calorie food nogo trials would elicit more activity in the prefrontal system than high-calorie food go trials; and (2) that high-calorie food go trials would elicit more activity in the habitual system than low-calorie food go trials. To rule out the possibility that the first contrast between nogo trials and go trials could partially be due to pre-potent go responses, a supplemental analysis was conducted by matching the number of go trials to the number of nogo trials. In this analysis, only the last go trial just prior to each nogo trial was included as an instance of the go trials. All other go trials were included as nuisance variables. This analysis generated similar findings. In all analyses, age and gender were included as covariates. Group images were evaluated with a height threshold of $\mathrm{Z}>2.3$ and a cluster probability of $\mathrm{p}<0.05$, corrected for whole-brain multiple comparisons based on Gaussian random field theory.

To correlate the nutrition data with brain activity, region of interests (ROI) were created from clusters of voxels with significant activation clusters in the voxelwise analyses. Analyses were performed by extracting parameter estimates (betas) of each event type from the fitted model and averaging across all voxels in the cluster for each participant/session. Percent signal changes were calculated using a method suggested by Mumford (http://mumford.fmripower.org/perchange_guide.pdf). Robust regression was used to minimize the impact of outliers in the behavioral data, using iteratively reweighted least squares implemented in the robustfit command in the MATLAB Statistics Toolbox [81]. Reported r-values reflect (non-robust) Pearson product-moment correlation values, whereas the reported $\mathrm{p}$-values and regression lines are based on the robust regression results [81]. This correlational analysis is highly relevant to determining whether increased activity in the prefrontal system on the high-calorie food nogo trials is due to simply an inhibition of a pre-potent response, or a greater difficulty in inhibiting a response to high-calorie foods.

\section{Results}

\section{Behavioral tasks and dietary intake}

Table 1 shows the mean, standard deviation, and range of the measures collected from the behavioral tasks and the 24-hour diet recall. Our results revealed normal intelligence (IQ) and working memory/executive functioning in our participants. BMI did not correlate with either IQ $(r=-.04, p=.83)$ or working memory capacity $(r=-.15, p=.46)$. Also, BMI did not correlate with age $(r=-.13, p=.50)$ or differ between genders $(t=1.67$, $p=.11)$. With respect to dietary intake, participants reported consuming $2.4 \pm 1.6$ servings/day/1ooo kcals of low-calorie foods (i.e., fruits and vegetables) and $1.8 \pm 1.3$ servings/day $/ 1000 \mathrm{kcals}$ of high-calorie foods (i.e., fatty foods and sugar-sweetened foods). Subjects showed a trend of consuming more servings/day/1000 kcals of low-calorie foods than high-calorie foods $(t(29)=$ 1.70, $p=.10$ ). The consumption of high-calorie foods (servings/day/1000 kcals) was independent of age $(r=.04$, $p=.84)$, BMI $(r=-.04, p=.84)$, and hunger rating $(r=-.15$, $p=.48)$. There were no gender differences with respect to IQ, SOPT scores, hunger rating, or consumption of highcalorie foods per $1000 \mathrm{kcal}$ (Table 1). However, there was a significant gender difference in consumption of low-calorie foods per $1000 \mathrm{kcal}(t(28)=2.76, p<.01)$, with females reporting more consumption of low-calorie foods per $1000 \mathrm{kcal}(3.0 \pm 1.6)$ than males $(1.6 \pm 1.1)$.

\section{Behavioral results in fMRI tasks}

Table 2 summarizes the major behavioral measures for the fMRI go/nogo tasks, including hit rates, false alarm rates, sensitivity index d', decision bias $\mathrm{C}$, and reaction

Table 2 Behavioral measures from the food-specific go/nogo task

\begin{tabular}{cccc}
\hline & LGo task & HGo task & t statistics \\
\hline Hits Rate & $.92 \pm .07$ & $.91 \pm .06$ & $\mathrm{t}=1.52, \mathrm{p}=.14$ \\
False Alarm Rate & $.18 \pm .11$ & $.13 \pm .09$ & $\mathrm{t}=3.03, \mathrm{p}=.005^{* *}$ \\
Go trial RT (ms) & $501.6 \pm 73.7$ & $484.2 \pm 65.2$ & $\mathrm{t}=-1.91, \mathrm{p}=.06$ \\
Nogo trial RT (ms) & $433.1 \pm 80.1$ & $419.4 \pm 80.7$ & $\mathrm{t}=-.80, \mathrm{p}=.43$ \\
$\mathbf{d}^{\prime}$ & $2.52 \pm .49$ & $2.58 \pm .58$ & $\mathrm{t}=-.64, \mathrm{p}=.53$ \\
$\mathbf{C}$ & $-.34 \pm .42$ & $-.10 \pm .39$ & $\mathrm{t}=-4.05, \mathrm{p}<.001^{* *}$ \\
\hline
\end{tabular}

** $\mathrm{p}<.01$ corrected for multiple comparison using Bonferroni correction. LGo Task low-calorie food go/high-calorie food nogo task, HGo Task high-calorie food go/low-calorie food nogo task, $R T$ reaction time. 
times for go trials and nogo trials (false alarm rates or inhibitory failures only). For each behavioral measure, paired t-tests were performed to test the difference between tasks (LGo vs HGo task). Analyses revealed that the false alarm rate in the LGo task was higher than in the HGo task $(t=3.03, p<.01)$. Similarly, decision bias $C$ in the LGo task was smaller than in the HGo task $(t=-4.05, p<.01)$. Both effects survived the Bonferroni correction for multiple comparisons. These results suggest that participants made more errors and had a harder time inhibiting responses to high-calorie food cues in the LGo task. Results also suggest that the reaction time for go trials tended to be longer in the LGo task than in the HGo task $(t=1.91, p=.06)$, although this effect did not survive the Bonferroni correction for multiple comparisons. This suggests that participants tended to more readily press the response button when go trials consisted of high-calorie food images in the HGo task. No other significant differences were found (all $p>.05$ ).

Finally, controlling for participants' age and gender, several correlations were significant among the behavioral measures. Reaction time for the go trials in the HGo task was negatively correlated with both BMI $(r=-.60, p<.01)$ and high-calorie food consumption $(r=-.50, p<.05)$, suggesting the habitual response to the high-calorie foods was greater for individuals with higher BMI, and individuals who reportedly consumed more high-calorie foods. The decision bias $\mathrm{C}$ for the LGo task negatively correlated with both BMI $(r=-.47, p<.05)$ and high-calorie food consumption $(r=-.49, p<.05)$, suggesting the inhibiting response to the high-calorie foods was more difficult for individuals with higher BMI and individuals who reported consuming more high-calorie food.

\section{fMRI data}

Table 3 summarized the fMRI results. First, imaging results suggested a significant main effect of Task in a few

Table 3 Summary of the fMRI results (factor analysis)

\begin{tabular}{|c|c|c|c|c|c|c|}
\hline Hemisphere & Brain region & Voxels & $\mathbf{x}$ & y & $\mathbf{z}$ & $\mathbf{Z}$ \\
\hline \multicolumn{7}{|c|}{ Main Effect of Task (Nogo > Go) } \\
\hline $\mathrm{L}$ & DLPFC/Insula & 280 & -32 & 24 & -8 & 3.56 \\
\hline $\mathrm{R}$ & Frontal Pole & 221 & 28 & 46 & 26 & 3.42 \\
\hline $\mathrm{R}$ & DLPFC/Insula & 218 & 32 & 26 & -14 & 3.12 \\
\hline$L$ & Frontal Pole & 99 & -22 & 58 & -6 & 3.25 \\
\hline$L / R$ & $\mathrm{ACC}$ & 92 & 4 & 44 & 4 & 2.85 \\
\hline \multicolumn{7}{|c|}{ Main Effect of Stimuli (High-calorie > Low-calorie Food Cues) } \\
\hline $\mathrm{L}$ & Occipital Pole & 182 & -6 & -100 & 4 & 3.28 \\
\hline \multicolumn{7}{|c|}{ Interaction between Task and Stimuli } \\
\hline & & None & & & & \\
\hline
\end{tabular}

$L$ Left, $R$ Right, ACC Anterior Cingulate Cortex, DLPFC Dorsolateral Prefrontal Cortex. brain regions, including bilateral frontal pole, bilateral dorsolateral prefrontal cortex (DLPFC) extending to the insular cortex, and anterior cingulate cortex (ACC). These brain regions were activated more during nogo trials than during go trials (Table 3 and Figure 2A). Second, the left occipital pole showed a significant main effect of stimuli with the high-calorie food pictures activating this region more than the low-calorie food pictures (Table 3 and Figure 2B). Third, no interaction effect between task and stimuli was found in any brain region.

Analyses comparing the high-calorie food go and nogo trials revealed that the neural systems referred to as the "reflective" system or prefrontal system [29,82] showed more activation during nogo trials than go trials, including bilateral activation of the DLPFC, insula, frontal pole, and ACC, and activation of the right superior frontal gyrus (Table 4 and Figure 2C). Supplemental analysis showed similar results when matching the number of go trials to the number of nogo trials. Further, of particular interest to us, ROI analysis suggested that the activation in the ACC region when comparing nogo trials to go trials (Figure $3 \mathrm{~A}, \mathrm{MNI}=4,44,4$ ) was negatively correlated with BMI (Figure 3B; $r=-.71, p<.01$ ) and high-calorie food consumption as measured by the 24-hour recall/NDSR (Figure 3C; $r=-.69, p<.01$ ). Also, females showed a trend of more activation in ACC than males when comparing nogo trials to go trials $(t=1.97, p=.06)$.

Results comparing go trials only revealed that the high-calorie food cues were associated with higher activity in the right striatum relative to low-calorie food cues (Table 4 and Figure 2D), a region belonging to what we have referred to as the "habitual" system. ROI analysis suggested that the degree of this increased activity in the right striatum (Figure $4 \mathrm{~A} ; \mathrm{MNI}=10,12,2$ ) was positively correlated with both BMI (Figure 4B; $r=.39, p<.05$ ) and level of actual high-calorie food consumption in real-life, assessed with the NDSR (Figure 4C; $r=.50, p<.01$ ). There was no significant difference between males and females in the activation of right striatum $(t=.49, p=.63)$.

\section{Discussion}

The present study used varieties of food specific go/nogo tasks to investigate the neural activity underlying response inhibition or the ability to control one's impulses when faced with images of high-calorie foods. Behaviorally, participants more readily pressed a response button when go trials signaled high-calorie foods during a highcalorie food go and low-calorie food nogo task (HGo task). We interpret these results to mean that the highcalorie food stimuli elicited more habitual responding and this response to high-calorie food stimuli was significantly greater for individuals with higher BMI, and also for those who consumed more high-calorie foods. 


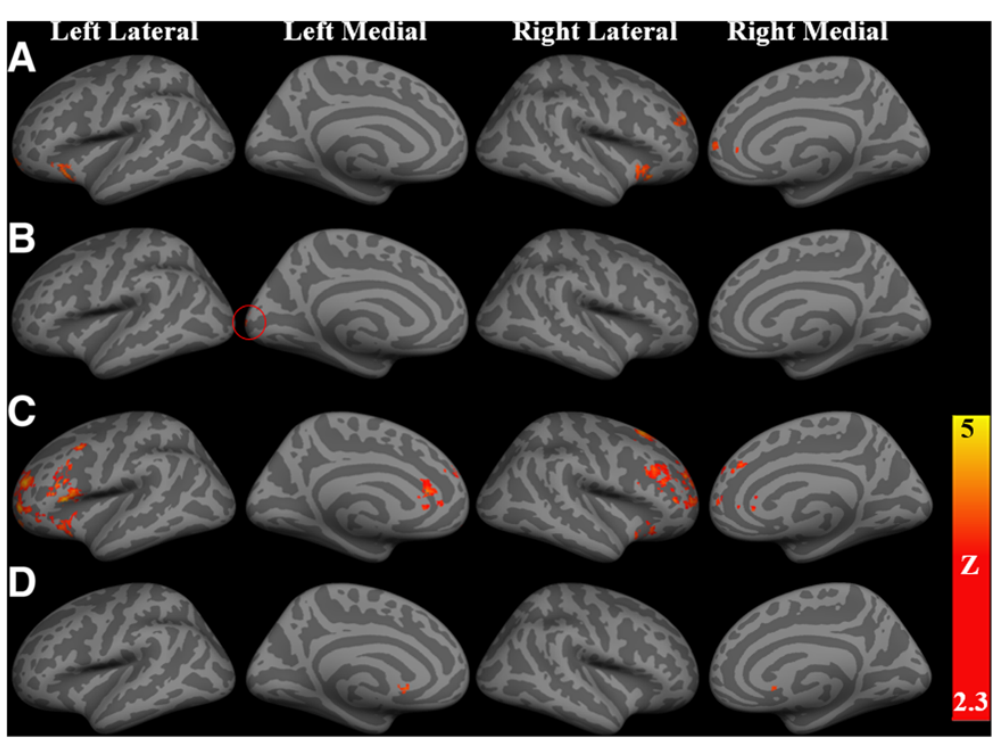

Figure 2 Summary of fMRI results rendered onto an averaged brain by FreeSurfer. A) Several brain regions showed a significant main effect of Task, including bilateral frontal pole, bilateral dorsolateral prefrontal cortical (DLPFC) extending to insular cortex, and anterior cingulate cortex (ACC). Nogo trials activated more than go trials in these regions. B) The left occipital pole (circled) showed a significant main effect of Stimuli with high-calorie food activated more than low-calorie food. C) The prefrontal system showed higher activation for high-calorie food nogo trials than high-calorie food go trials, including bilateral DLPFC, insula, frontal pole, ACC and right superior frontal gyrus. D) The "habitual" system (right striatum) showed higher activity in high-calorie food go trials relative to low-calorie food go trials. No brain region showed an interaction effect between Task and Stimuli.

Furthermore, it was more difficult for participants to inhibit their response to high-calorie foods in the LGo task (low-calorie food go and high-calorie food nogo) than low-calorie foods in the HGo task. Similar to habitual responding to high-calorie foods, the ability to inhibit responding to high-calorie foods was more difficult for individuals with higher BMI and individuals who reported consuming more high-calorie foods. The fMRI findings further supported our hypotheses that (1) the habitual system was activated more in response to highcalorie food go trials than low-calorie food go trials, and its activation correlated with participants' BMI as well as their consumption of high-calorie foods; and (2) the prefrontal system was more engaged during nogo trials than the go trials, and its activation was inversely correlated with BMI and high-calorie food consumption.

The habitual system observed in the present study was located in the right striatum, which is a dopamine-dependent neural system critical for the incentive motivational effects of a variety of non-natural rewards (e.g., psychoactive drugs) and natural rewards (e.g., food) [32,83-89]. This is also the neural system that has been argued to be responsible for the transfer of reward seeking from controlled to automatic and habitual behaviors [32]. The so-called "habitual" system has

Table 4 fMRI results related to habitual and prefrontal system

\begin{tabular}{|c|c|c|c|c|c|c|}
\hline Hemisphere & Brain region & Voxels & $\mathrm{x}$ & $y$ & $z$ & $\mathrm{Z}$ \\
\hline \multicolumn{7}{|c|}{ Nogo > Go (High-calorie Food Cues Only) } \\
\hline$L / R$ & ACC extending to left Frontal Pole & 1458 & -30 & 50 & 2 & 3.50 \\
\hline L & DLPFC/Insula & 1220 & -40 & 20 & 8 & 3.73 \\
\hline $\mathrm{R}$ & DLPFC & 840 & 44 & 18 & 26 & 3.24 \\
\hline L & Frontal Pole & 308 & -24 & 62 & -6 & 3.76 \\
\hline $\mathrm{R}$ & Frontal Pole & 214 & 34 & 42 & 4 & 3.47 \\
\hline $\mathrm{R}$ & Superior Frontal Gyrus & 205 & 18 & 22 & 58 & 3.56 \\
\hline $\mathrm{R}$ & Insula/Orbital Frontal Cortex & 185 & 36 & 28 & -22 & 2.93 \\
\hline \multicolumn{7}{|c|}{ High-calorie > Low-calorie Food Cues (Go Trials Only) } \\
\hline $\mathrm{R}$ & Striatum & 60 & 10 & 12 & 2 & 2.74 \\
\hline
\end{tabular}

L Left, $R$ Right, ACC Anterior Cingulate Cortex, DLPFC Dorsolateral Prefrontal Cortex. 

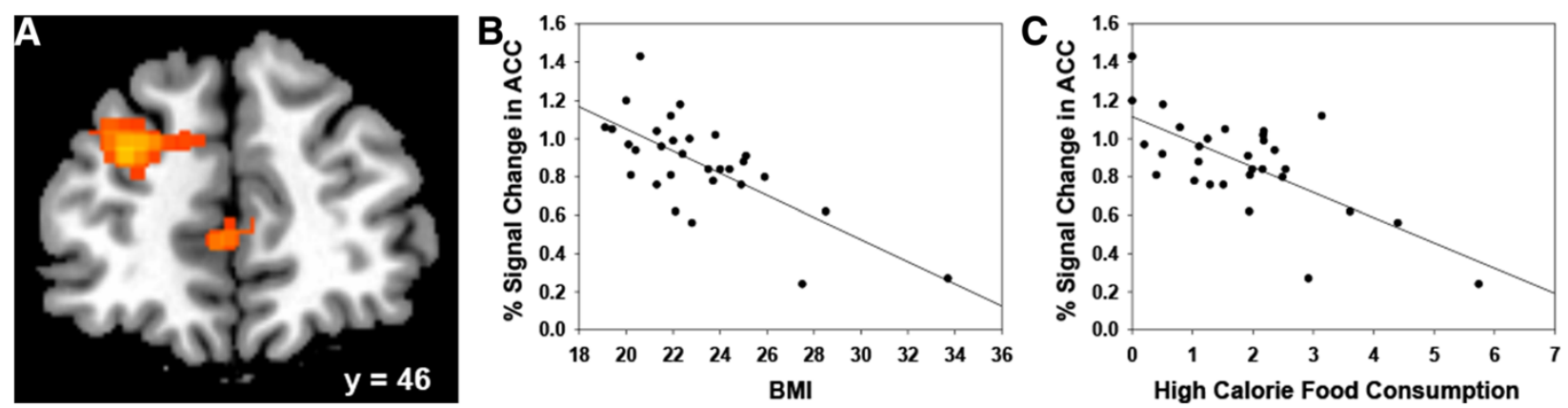

Figure 3 Activity of the ACC was inversely correlated with both BMI and NDSR. A) The ACC was activated when comparing nogo trials to go trials. Slices are displayed in radiological view (right is on the viewer's left). B) Scatter plot showed the correlation between ACC activation and BMI. C) Scatter plot showed the correlation between ACC activation and high-calorie food consumption tested by NDSR. ACC: anterior cingulate cortex; BMI: body mass index; NDSR: nutrition data system for research.

been shown to become hyperactive and to exaggerate the incentive value of rewards in individuals with substance abuse problems [28]. Given the similarities between the responses to images of high-calorie foods we observed in this study and to those responses others have observed among substance (ab)users to drug-related cues, this study adds to the evidence supporting a food-addiction model of obesity. Further, our findings are consistent with previous studies that have demonstrated that food cues (such as the sight or smell of food) engage the mesolimbic dopamine-striatal system, especially in obese individuals [13,16,46-52,90,91]. For example, emerging findings suggest that the fat and sugar present in high-calorie foods may particularly engage these dopamine-dependent reward systems [13] and motivate food seeking behaviors [16,46,61]. fMRI studies also show that compared to non-food-related pictures, food-related pictures activate the striatum [92] in healthy individuals. Consistent with this finding, we observed right striatum activation when responses to high-calorie food pictures were compared to responses to low-calorie food pictures, although previous studies showed that the dorsal striatum is not strictly dedicated to habit behaviors, and that it can be involved in decision-making [28,82,93-95]. Animal studies also have shown that direct pharmacological activation of the striatum, amygdalo-hypothalamic circuit produced hyperphagia and increased preferentially the intake of foods high in fat and sugar, even in animals fed beyond apparent satiety [96].

Additionally, we found that activation of the "habitual" system correlated with both BMI and daily consumption of high-calorie foods. However, we did not find a significant correlation between BMI and high-calorie food consumption behaviorally. The present study consisted of a sample of mostly normal-weight participants, and this lack of variance in BMI might preclude replicating behavioral findings observed in other studies between obese and normal weight individuals in the consumption of high-calorie foods [92,97]. Nevertheless, the significant correlation between brain activation and BMI, as well as daily consumption of high-calorie foods, is consistent with several lines of evidence that suggest that highly palatable food may induce greater incentive values in obese individuals compared to normal controls [92,97]. Behavioral studies also show that overweight children
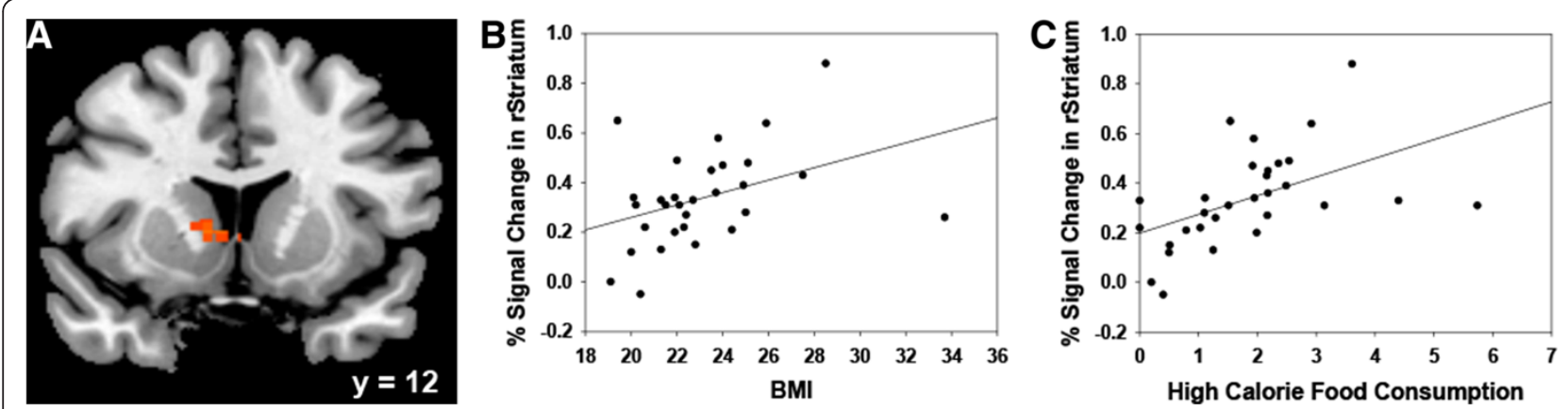

Figure 4 Activity of the striatum was positively correlated with both BMI and NDSR. A) The activation of the right striatum was revealed when comparing snack go trials to vegetable go trials. Slices are displayed in radiological view (right is on the viewer's left). B) Scatter plot showed the correlation between the right striatum activation and BMI. C) Scatter plot showed the correlation between the right striatum activation and high-calorie food consumption tested by NDSR. BMI: body mass index; NDSR: nutrition data system for research. 
indicate that high-calorie food consumption (e.g., snack foods) is more reinforcing than what their leaner peers indicate [97]. The relative reinforcing value of food versus two non-food alternatives (e.g., time spent playing a handheld video game or time spent reading magazines or completing word searches or mazes) is also high in overweight children, and relatively low in non-overweight children [97]. Using fMRI, Beaver and colleagues [92] reported that individual variation in trait reward sensitivity correlated highly with activation to images of highly palatable, appetizing foods (e.g., chocolate, ice cream) in a frontostriatal-amygdala-midbrain network in healthy volunteers. Beaver and colleagues' study, together with our findings in adolescents and young adults, suggest that the habitual system may be hyper-active when facing highly palatable and high-calorie foods, such that it begins to exaggerate the incentive impact of food reward, contributing to overweight and obesity.

This study further showed that the nogo trials relative go trials engaged several regions implicated in the prefrontal system, including the ACC and bilateral frontal pole, when participants performed the food-specific go/ nogo task. Implicated regions of the prefrontal system including the ACC, frontal poles and DLPFC help to control basic impulses and allow more flexible pursuit of long-term goals $[98,99]$. The prefrontal system is important for good decision-making and inhibitory control. Several studies suggest that the cognitive or regulatory control of food intake is in the prefrontal system [100-103], and this system shows an altered response to food cues $[59,60]$. For example, one study showed that lean individuals had greater neural activity than obese individuals in the prefrontal cortex when inhibiting food consumption due to satiation [101]. Consistent with our findings, another study found significant increases in the activation of the OFC in response to high-calorie food images, but not to low-calorie food images, suggesting change in reward evaluation and response inhibition in reaction to fatty and unhealthy food images [102]. Further, the prefrontal system appears to be hypo-active for those who consume more high-calorie food daily as reflected by the inverse correlation between ACC activation and high-calorie food consumption.

Gender differences in decision-making and inhibitory control processes have been observed with respect to various appetitive/addictive behaviors [104-113]. For example, males have been found to be more likely than females to engage in risky behaviors [110], and males appear to be less able to control inappropriate behaviors than females [111]. One study found that after consumption of the same amount of alcohol, the capacity to inhibit or control behavior was less impaired in women relative to men [112]. Another study found marked gender differences in inhibitory control with respect to consumption of sugarsweetened snacks on food-cued and generic go/nogo tasks [113]. Inhibitory problems measured with these tasks were strong correlates of sweet snack consumption in males, but not in females. Similar to these findings, the present study found that females consumed more low-calorie food than males, which might be related to the notion that they have a stronger neural system for impulse control towards highcalorie food, and this is supported by the finding of higher ACC activation in the food-specific go/nogo task.

The present study has several limitations to be addressed in future studies. First, we did not find a correlation between BMI and high-calorie food consumption. This may simply be due to measurement error that is inherent in a single dietary assessment or possibly due to variations in developmental status (e.g., continued maturation, particularly among the young men) [114-118] or physical activity energy expenditure in this young adult population. Second, participants were provided instructions for eating prior to the study session. Their fed state was confirmed using self-reported, perceived hunger status rather than providing a standardized meal before the scan. Although providing a standardized meal could minimize the influence of hunger status on task performance $[119,120]$, it could also introduce a confounding factor; that is, "liking" or "disliking" the meal. Examining potential differences in neural responses due to eating standardized versus normal meals prior to scanning should be conducted in future studies. Third, we did not measure levels of appetite hormones prior to scanning in this study. Again, this would be an important question to address in future studies given the available evidence showing that these hormones are important to decision making around diet and nutrition [121]. Another important aspect of inhibitory control not addressed in the current study is the role of cognitive restraint that participants exert over eating behavior. Previous studies have shown that the cognitive restraint participants exert over eating behavior influences brain activity during an inhibitory task [122,123]. Though we did not explicitly assess cognitive dietary restraint or weight control status in this sample, it is an important issue that deserves attention in future research. Finally, our subjects did not consume real food while in the scanner; instead, they viewed images of food. This design could potentially reduce the ecological validity of the task, although numerous fMRI studies conducted to date have used food images instead of real food to measure food choice $[11,17,18,47,92,124-126]$.

\section{Conclusion}

In conclusion, the present study among adolescents and young adults used food-specific go/nogo tasks to investigate brain activity underlying self-control when faced 
with tempting food choices. Results confirmed our hypotheses regarding two key neural systems involved in decisions to seek reward from appetizing foods: a hyperactive habitual system and a hypo-active prefrontal system in this population. Though the cross-sectional design does not permit inferences regarding whether the inhibitory control deficits and hyper-responsivity of reward regions are individual vulnerability factors for overeating or results of habitual overeating. Our results shed light on the neural basis of one's loss of ability to self-control when faced with tempting food choices, which could potentially contribute to the development of intervention strategies aimed at reducing the consumption of high-calorie foods. Such intervention strategies are important in order to help reduce the incidence of obesity as adolescents and young adults approach adulthood, thereby reducing the future risk of obesity-related chronic diseases and cancers.

\section{Competing interests}

The authors declare that they have no competing interests.

\section{Authors' contribution}

Conceived and designed the experiments: QH LX GX SW SLA AB. Performed the experiments: QH LX GX. Analyzed the data: QH LX SMS. Contributed reagents/materials/analysis tools: QH GX. Wrote the paper: QH LX SAL SMS $A B$. All authors read and approved the final manuscript.

\section{Acknowledgments}

This research was supported by research grants from National Institute on Drug Abuse (NIDA) R01DA023051, National Cancer Institute (NCI) R01CA152062, National Heart, Lung, \& Blood Institute and the National Institute of Child Health \& Human Development (U01HL097839), Fundamental Research Funds for the Central Universities (SWU1409349), and Open Research Fund of the National Key Laboratory of Cognitive Neuroscience and Learning (CNLZD1306). We would also like to thank Alexandra Hollihan and Stephanie Castillo who helped with the data collection.

\section{Author details}

${ }^{1}$ Faculty of Psychology, Southwest University, Beibei, Chongqing, China. ${ }^{2}$ Brain and Creativity Institute and Department of Psychology, University of Southern California, Los Angeles, CA, USA. ${ }^{3}$ National Key Laboratory of Cognitive Neuroscience and Learning \& IDG/McGovern Institute for Brain Research, Beijing Normal University, Beijing, China. ${ }^{4}$ Department of Special Education and Counselling, The Hong Kong Institute of Education, Hong Kong, China. ${ }^{5}$ School of Community and Global Health, Claremont Graduate University, Claremont, CA, USA. ${ }^{6}$ Department of Behavioral Science, The University of Texas MD Anderson Cancer Center, Houston, TX, USA.

Received: 1 May 2014 Accepted: 12 September 2014

Published: 16 September 2014

\section{References}

1. Flegal KM, Carroll MD, Ogden CL, Johnson CL: Prevalence and trends in obesity among US adults, 1999-2000. JAMA: J Am Med Assoc 2002, 288:1723-1727.

2. Renehan AG, Tyson M, Egger M, Heller RF, Zwahlen M: Body-mass index and incidence of cancer: a systematic review and meta-analysis of prospective observational studies. Lancet 2008, 371:569-578.

3. Janssen I, Katzmarzyk P, Boyce W, Vereecken C, Mulvihill C, Roberts C, Currie C, Pickett W: Comparison of overweight and obesity prevalence in school-aged youth from 34 countries and their relationships with physical activity and dietary patterns. Obes Rev 2005, 6:123-132.

4. Loos RJ, Yeo GS: The bigger picture of FTO [mdash] the first GWAS-identified obesity gene. Nat Rev Endocrinol 2014, 10:51-61.

5. Zhao J, Bradfield JP, Zhang H, Sleiman PM, Kim CE, Glessner JT, Deliard S, Thomas KA, Frackelton EC, Li M: Role of BMI-associated loci identified in
GWAS meta-analyses in the context of common childhood obesity in European Americans. Obesity 2011, 19:2436-2439.

6. Yang X, Deignan JL, Qi H, Zhu J, Qian S, Zhong J, Torosyan G, Majid S, Falkard B, Kleinhanz RR: Validation of candidate causal genes for obesity that affect shared metabolic pathways and networks. Nat Genet 2009, 41:415-423

7. Martínez JA, Cordero P, Campión J, Milagro Fl: Interplay of early-life nutritional programming on obesity, inflammation and epigenetic outcomes. Proc Nutr Soc 2012, 71:276-283.

8. Martinelli CE, Keogh JM, Greenfield JR, Henning E, van der Klaauw AA, Blackwood A, O'Rahilly S, Roelfsema F, Camacho-Hübner C, Pijl H: Obesity due to melanocortin 4 receptor (MC4R) deficiency is associated with increased linear growth and final height, fasting hyperinsulinemia, and incompletely suppressed growth hormone secretion. J Clinical Endocrinol Metabol 2011, 96:E181-E188.

9. Gutierrez-Aguilar R, Kim DH, Woods SC, Seeley RJ: Expression of New loci associated with obesity in diet-induced obese rats: from genetics to physiology. Obesity 2012, 20:306-312.

10. Davis C, Patte K, Levitan R, Reid C, Tweed S, Curtis C: From motivation to behaviour: a model of reward sensitivity, overeating, and food preferences in the risk profile for obesity. Appetite 2007, 48:12-19.

11. Stice E, Spoor S, Bohon C, Veldhuizen M, Small D: Relation of reward from food intake and anticipated food intake to obesity: a functional magnetic resonance imaging study. J Abnorm Psychol 2008, 117:924-935.

12. Small DM: Individual differences in the neurophysiology of reward and the obesity epidemic. Int J Obes 2009, 33:S44-S48.

13. Killgore WD, Young AD, Femia LA, Bogorodzki P, Rogowska J, YurgelunTodd DA: Cortical and limbic activation during viewing of high-versus low-calorie foods. Neuroimage 2003, 19:1381-1394.

14. Killgore WD, Yurgelun-Todd DA: Body mass predicts orbitofrontal activity during visual presentations of high-calorie foods. Neuroreport 2005, 16:859-863.

15. DelParigi A, Chen K, Salbe AD, Reiman EM, Tataranni PA: Sensory experience of food and obesity: a positron emission tomography study of the brain regions affected by tasting a liquid meal after a prolonged fast. Neuroimage 2005, 24:436-443.

16. DelParigi A, Chen K, Salbe A, Hill J, Wing R, Reiman E, Tataranni P: Successful dieters have increased neural activity in cortical areas involved in the control of behavior. Int J Obes 2006, 31:440-448.

17. Pelchat ML, Johnson A, Chan R, Valdez J, Ragland JD: Images of desire: food-craving activation during fMRI. Neuroimage 2004, 23:1486-1493.

18. Batterink L, Yokum S, Stice E: Body mass correlates inversely with inhibitory control in response to food among adolescent girls: an fMRI study. Neuroimage 2010, 52:1696-1703.

19. Ng J, Stice E, Yokum S, Bohon C: An fMRI study of obesity, food reward, and perceived caloric density. Does a low-fat label make food less appealing? Appetite 2011, 57:65-72.

20. Kelley $A E$, Berridge $K C$ : The neuroscience of natural rewards: relevance to addictive drugs. J Neurosci 2002, 22:3306-3311.

21. Rolls E: Understanding the mechanisms of food intake and obesity. Obes Rev 2007, 8:67-72.

22. Trinko R, Sears RM, Guarnieri DJ, DiLeone RJ: Neural mechanisms underlying obesity and drug addiction. Physiol Behav 2007, 91:499-505.

23. Volkow ND, Wang GJ, Fowler JS, Telang F: Overlapping neuronal circuits in addiction and obesity: evidence of systems pathology. Phil Trans Royal Soc B: Biol Sci 2008, 363:3191-3200.

24. Johnson PM, Kenny PJ: Dopamine D2 receptors in addiction-like reward dysfunction and compulsive eating in obese rats. Nat Neurosci 2010, 13:635-641.

25. Saelens BE, Epstein LH: Reinforcing value of food in obese and non-obese women. Appetite 1996, 27:41-50.

26. Smith JA, Epstein LH: Behavioral economic analysis of food choice in obese children. Appetite 1991, 17:91-95.

27. Vicennati V, Pasqui F, Cavazza C, Garelli S, Casadio E, di Dalmazi G, Pagotto $U$, Pasquali R: Cortisol, energy intake, and food frequency in overweight/ obese women. Nutrition 2011, 27:677-680.

28. Bechara A: Decision making, impulse control and loss of willpower to resist drugs: a neurocognitive perspective. Nat Neurosci 2005, 8:1458-1463.

29. Noël X, Brevers D, Bechara A: A neurocognitive approach to understanding the neurobiology of addiction. Curr Opin Neurobio/ 2013, 23:632-638. 
30. Ames SL, Grenard JL, He Q, Stacy AW, Wong SW, Xiao L, Xue G, Bechara A: Functional imaging of an alcohol-implicit association test (IAT). Addict Biol 2014, 19:467-481.

31. Ames SL, Grenard JLW Stacy A, Xiao L, He Q, Wong SW, Xue GW, Wiers R, Bechara A: Functional imaging of implicit marijuana associations during performance on an implicit association test (IAT). Behav Brain Res 2013. 256:494-502.

32. Everitt $B$, Robbins T: Neural systems of reinforcement for drug addiction: from actions to habits to compulsion. Nat Neurosci 2005, 8:1481-1489.

33. Everitt BJ, Parkinson JA, Olmstead MC, Arroyo M, Robledo P, Robbins TW: Associative processes in addiction and reward the role of amygdala-ventral striatal subsystems. Ann N Y Acad Sci 2006, 877:412-438.

34. Aron AR, Robbins TW, Poldrack RA: Inhibition and the right inferior frontal cortex: one decade on. Trends Cogn Sci 2014, 18:177-185.

35. Van Holst RJ, Van Holstein M, Van Den Brink W, Veltman DJ, Goudriaan AE: Response inhibition during cue reactivity in problem gamblers: an fMRI study. Plos One 2012, 7:e30909.

36. Gläscher J, Adolphs R, Damasio H, Bechara A, Rudrauf D, Calamia M, Paul LK, Tranel D: Lesion mapping of cognitive control and value-based decision making in the prefrontal cortex. Proc Natl Acad Sci 2012, 109:14681-14686.

37. Goldstein RZ, Volkow ND: Dysfunction of the prefrontal cortex in addiction: neuroimaging findings and clinical implications. Nat Rev Neurosci 2011, 12:652-669

38. Steinbeis N, Bernhardt BC, Singer T: Impulse control and underlying functions of the left DLPFC mediate age-related and age-independent individual differences in strategic social behavior. Neuron 2012, 73:1040-1051.

39. Aron A, Robbins T, Poldrack R: Inhibition and the right inferior frontal cortex. Trends Cogn Sci 2004, 8:170-177.

40. Naqvi NH, Bechara A: The hidden island of addiction: the insula. Trends Neurosci 2009, 32:56-67.

41. Craig AD: How do you feel? Interoception: the sense of the physiological condition of the body. Nat Rev Neurosci 2002, 3:655-666.

42. Craig AD: Interoception: the sense of the physiological condition of the body. Curr Opin Neurobiol 2003, 13:500-505.

43. Craig AD: How do you feel-now? The anterior insula and human awareness. Nat Rev Neurosci 2009, 10:59-70.

44. Craig AD, Chen K, Bandy D, Reiman EM: Thermosensory activation of insular cortex. Nat Neurosci 2000, 3:184-190.

45. Paulus MP, Stein MB: An insular view of anxiety. Biol Psychiatry 2006, 60:383-387.

46. Grill HJ, Skibicka KP, Hayes MR: Imaging obesity: fMRI, food reward, and feeding. Cell Metab 2007, 6:423-425.

47. Stoeckel LE, Weller RE, 3rd Cook E, Twieg DB, Knowlton RC, Cox JE: Widespread reward-system activation in obese women in response to pictures of high-calorie foods. Neuroimage 2008, 41:636-647.

48. Demos KE, Kelley WM, Heatherton TF: Dietary restraint violations influence reward responses in nucleus accumbens and amygdala. J Cogn Neurosci 2011, 23:1952-1963.

49. Small DM: Toward an understanding of the brain substrates of reward in humans. Neuron 2002, 33:668-671.

50. Schur E, Kleinhans N, Goldberg J, Buchwald D, Schwartz M, Maravilla K: Activation in brain energy regulation and reward centers by food cues varies with choice of visual stimulus. Int J Obes 2009, 33:653-661.

51. Simmons WK, Martin A, Barsalou LW: Pictures of appetizing foods activate gustatory cortices for taste and reward. Cereb Cortex 2005, 15:1602-1608.

52. Passamonti $L$, Rowe JB, Schwarzbauer $C$, Ewbank MP, von dem Hagen $E$, Calder AJ: Personality predicts the brain's response to viewing appetizing foods: the neural basis of a risk factor for overeating. J Neurosci 2009, 29:43-51.

53. Bruce AS, Lepping RJ, Bruce JM, Cherry JBC, Martin LE, Davis AM, Brooks WM, Savage CR: Brain responses to food logos in obese and healthy weight children. J Pediatr 2013, 162:759-764.

54. García-García I, Jurado MÁ, Garolera M, Segura B, Sala-Llonch R, Marqués-Iturria I, Pueyo R, Sender-Palacios MJ, Vernet-Vernet M, Narberhaus A: Alterations of the salience network in obesity: a resting-state fMRI study. Hum Brain Mapp 2013, 34:2786-2797.

55. Martens MJ, Born JM, Lemmens SG, Karhunen L, Heinecke A, Goebel R, Adam TC, Westerterp-Plantenga MS: Increased sensitivity to food cues in the fasted state and decreased inhibitory control in the satiated state in the overweight. Am J Clinical Nutri 2013, 97:471-479.

56. Tsao S, Adam TC, Goran MI, Singh M: Application of fMRI to obesity research: differences in reward pathway activation measured with fMRI
BOLD during visual presentation of high and low calorie foods. In SPIE Medical Imaging: 4-9 February 2012; San Diego. Edited by Molthen RC, Weaver JB, SPIE; 2012:83170B-1-83170B-6.

57. Cornier M-A, McFadden KL, Thomas EA, Bechtell IL, Eichman LS, Bessesen DH, Tregellas JR: Differences in the neuronal response to food in obesity-resistant as compared to obesity-prone individuals. Physiol Behav 2013, 110:122-128.

58. Murdaugh DL, Cox JE, III Cook EW, Weller RE: fMRI reactivity to high-calorie food pictures predicts short-and long-term outcome in a weight-loss program. Neuroimage 2012, 59:2709-2721.

59. Hare TA, Camerer CF, Rangel A: Self-control in decision-making involves modulation of the vmPFC valuation system. Science 2009, 324:646-648.

60. Hare TA, Malmaud J, Rangel A: Focusing attention on the health aspects of foods changes value signals in vmPFC and improves dietary choice. J Neurosci 2011, 31:11077-11087.

61. Geliebter A, Ladell T, Logan M, Schweider T, Sharafi M, Hirsch J: Responsivity to food stimuli in obese and lean binge eaters using functional MRI. Appetite 2006, 46:31-35.

62. Rothemund Y, Preuschhof C, Bohner G, Bauknecht H-C, Klingebiel R, Flor H, Klapp BF: Differential activation of the dorsal striatum by high-calorie visual food stimuli in obese individuals. Neuroimage 2007, 37:410-421.

63. Tomasi D, Volkow ND: Striatocortical pathway dysfunction in addiction and obesity: differences and similarities. Crit Rev Biochem Mol Biol 2013, 48:1-19.

64. García-García I, Narberhaus A, Marqués-Iturria I, Garolera M, Rădoi A, Segura B, Pueyo R, Ariza M, Jurado M: Neural responses to visual food cues: insights from functional magnetic resonance imaging. Eur Eat Disord Rev 2013, 21:89-98.

65. Brooks SJ, Cedernaes J, Schiöth HB: Increased prefrontal and parahippocampal activation with reduced dorsolateral prefrontal and insular cortex activation to food images in obesity: a meta-analysis of fMRI studies. Plos One 2013, 8:e60393.

66. Giedd J, Blumenthal J, Jeffries N, Castellanos F, Liu H, Zijdenbos A, Paus T, Evans A, Rapoport J: Brain development during childhood and adolescence: a longitudinal MRI study. Nat Neurosci 1999, 2:861-862.

67. Reiss AL, Abrams MT, Singer HS, Ross JL, Denckla MB: Brain development, gender and IQ in children A volumetric imaging study. Brain 1996, 119:1763-1774.

68. Sowell ER, Peterson BS, Thompson PM, Welcome SE, Henkenius AL, Toga AW: Mapping cortical change across the human life span. Nat Neurosci 2003, 6:309-315.

69. Anding JD, Suminski RR, Boss L: Dietary intake, body mass index, exercise, and alcohol: are college women following the dietary guidelines for Americans? J Am Coll Heal 2001, 49:167-171.

70. Wechsler D: Wechsler Abbreviated Scale of Intelligence. New York, NY: The Psychological Corporation: Harcourt Brace \& Company; 1999.

71. Cragg L, Nation K: Self-ordered pointing as a test of working memory in typically developing children. Memory 2007, 15:526-535.

72. Feskanich D, Sielaff BH, Chong K, Buzzard IM: Computerized collection and analysis of dietary intake information. Comput Methods Prog Biomed 1989, 30:47-57.

73. Johnson RK, Driscoll P, Goran Ml: Comparison of multiple-pass 24-hour recall estimates of energy intake with total energy expenditure determined by the doubly labeled water method in young children. J Am Diet Assoc 1996, 96:1140-1144

74. Wang $Y$, Jahns L, Tussing-Humphreys L, Xie B, Rockett H, Liang H, Johnson L: Dietary intake patterns of low-income urban African American adolescents. J Am Diet Assoc 2010, 110:1340-1345

75. Jenkinson M, Smith S: A global optimisation method for robust affine registration of brain images. Med Image Anal 2001, 5:143-156.

76. Andersson JLR, Jenkinson M, Smith S: Non-linear registration, aka spatial normalisation. FMRIB technical report TR07JA2 from www.fmrib.ox.ac.uk/ analysis/techrep/tr07ja2/tr07ja2.pdf 2007

77. Andersson JLR, Jenkinson M, Smith S: Non-linear optimisation. FMRIB technical report TR07JA1 from www.fmrib.ox.ac.uk/analysis/techrep/tr07ja1/ tr07ja1.pdf 2007

78. Woolrich MW, Behrens TEJ, Beckmann CF, Jenkinson M, Smith SM: Multilevel linear modelling for FMRI group analysis using Bayesian inference. Neuroimage 2004, 21:1732-1747.

79. Beckmann CF, Jenkinson M, Smith SM: General multilevel linear modeling for group analysis in FMRI. Neuroimage 2003, 20:1052-1063. 
80. Woolrich M: Robust group analysis using outlier inference. Neuroimage 2008, 41:286-301.

81. Tom SM, Fox CR, Trepel C, Poldrack RA: The neural basis of loss aversion in decision-making under risk. Science 2007, 315:515-518.

82. Bechara A, Damasio AR: The somatic marker hypothesis: a neural theory of economic decision. Games Econom Behav 2005, 52:336-372.

83. Wise RA, Rompré PP: Brain dopamine and reward. Annu Rev Psychol 1989, 40:191-225.

84. Stewart J, De Wit H, Eikelboom R: Role of unconditioned and conditioned drug effects in the self-administration of opiates and stimulants. Psychol Rev 1984, 91:251-268.

85. Robinson TE, Berridge KC: Incentive-sensitization and addiction. Addiction 2001, 96:103-114.

86. Koob GF, Volkow ND: Neurocircuitry of addiction. Neuropsychopharmacology 2010, 35:217-238.

87. Balleine BW, Delgado MR, Hikosaka O: The role of the dorsal striatum in reward and decision-making. J Neurosci 2007, 27:8161-8165.

88. Di Chiara G, Bassareo V, Fenu S, De Luca MA, Spina L, Cadoni C, Acquas E, Carboni $E$, Valentini $V$, Lecca $D$ : Dopamine and drug addiction: the nucleus accumbens shell connection. Neuropharmacology 2004, 47:227-241.

89. Di Chiara G, Bassareo V: Reward system and addiction: what dopamine does and doesn't do. Curr Opin Pharmacol 2007, 7:69-76.

90. Small DM, Veldhuizen MG, Felsted J, Mak YE, McGlone F: Separable substrates for anticipatory and consummatory food chemosensation. Neuron 2008, 57:786-797.

91. Frank GK, Wagner A, Achenbach S, McConaha C, Skovira K, Aizenstein H, Carter CS, Kaye WH: Altered brain activity in women recovered from bulimic-type eating disorders after a glucose challenge: a pilot study. Int J Eat Disord 2006, 39:76-79.

92. Beaver JD, Lawrence AD, van Ditzhuijzen J, Davis MH, Woods A, Calder AJ: Individual differences in reward drive predict neural responses to images of food. J Neurosci 2006, 26:5160-5166.

93. Ding L, Gold Jl: Separate, causal roles of the caudate in saccadic choice and execution in a perceptual decision task. Neuron 2012, 75:865-874.

94. Gluth S, Rieskamp J, Büchel C: Deciding when to decide: time-variant sequential sampling models explain the emergence of value-based decisions in the human brain. J Neurosci 2012, 32:10686-10698.

95. Li X, Lu Z-L, D'Argembeau A, Ng M, Bechara A: The lowa gambling task in fMRI images. Hum Brain Mapp 2009, 31:410-423.

96. Kelley AE, Baldo BA, Pratt WE, Will MJ: Corticostriatal-hypothalamic circuitry and food motivation: integration of energy, action and reward. Physiol Behav 2005, 86:773-795.

97. Temple JL, Legierski CM, Giacomelli AM, Salvy S-J, Epstein LH: Overweight children find food more reinforcing and consume more energy than do nonoverweight children. Am J Clinical Nutri 2008, 87:1121-1127.

98. Fellows LK, Farah MJ: Is anterior cingulate cortex necessary for cognitive control? Brain 2005, 128:788-796.

99. Wheeler EZ, Fellows LK: The human ventromedial frontal lobe is critical for learning from negative feedback. Brain 2008, 131:1323-1331.

100. Grabenhorst F, Rolls ET, Parris BA: From affective value to decision-making in the prefrontal cortex. Eur J Neurosci 2008, 28:1930-1939.

101. Del Parigi A, Gautier J-F, Chen K, Salbe AD, Ravussin E, Reiman E, Tataranni PA Neuroimaging and obesity: mapping the brain responses to hunger and satiation in humans using positron emission tomography. Ann N Y Acad Sci 2002, 967:389-397.

102. Killgore WD, Yurgelun-Todd DA: Developmental changes in the functional brain responses of adolescents to images of high and low-calorie foods. Dev Psychobiol 2005, 47:377-397.

103. Davis C, Patte K, Curtis C, Reid C: Immediate pleasures and future consequences. A neuropsychological study of binge eating and obesity. Appetite 2010, 54:208-213.

104. Atalayer D, Pantazatos SP, Gibson CD, McOuatt H, Puma L, Astbury NM, Geliebter A: Sexually dimorphic functional connectivity in response to high vs. low energy-dense food cues in obese humans: An fMRI study. Neuroimage 2014, 100:405-413.

105. Killgore WDS, Yurgelun-Todd DA: Sex differences in cerebral responses to images of high versus low-calorie food. Neuroreport 2010, 21:354-358. 310.1097/WNR.1090b1013e32833774f32833777.

106. Cornier M-A, Salzberg AK, Endly DC, Bessesen DH, Tregellas JR: Sex-based differences in the behavioral and neuronal responses to food. Physiol Behav 2010, 99:538-543.
107. Mueller K, Anwander A, Möller HE, Horstmann A, Lepsien J, Busse F, Mohammadi S, Schroeter ML, Stumvoll M, Villringer A, Pleger B: Sex-dependent influences of obesity on cerebral white matter investigated by diffusiontensor imaging. Plos One 2011, 6:e18544.

108. Killgore WDS, Weber M, Schwab ZJ, Kipman M, DelDonno SR, Webb CA, Rauch SL: Cortico-limbic responsiveness to high-calorie food images predicts weight status among women. Int J Obes 2013, 37:1435-1442.

109. Geliebter A, Pantazatos SP, McOuatt H, Puma L, Gibson CD, Atalayer D: Sex-based $\mathrm{fMRI}$ differences in obese humans in response to high vs. low energy food cues. Behav Brain Res 2013, 243:91-96.

110. Becker B, Perry N, Westenbroek C: Sex differences in the neural mechanisms mediating addiction: a new synthesis and hypothesis. Bio/ Sex Differ 2012, 3:14.

111. Yuan J, He Y, Qinglin Z, Chen A, Li H: Gender differences in behavioral inhibitory control: ERP evidence from a two-choice oddball task. Psychophysiology 2008, 45:986-993.

112. Fillmore MT, Weafer J: Alcohol impairment of behavior in men and women. Addiction 2004, 99:1237-1246.

113. Ames SL, Kisbu-Sakarya Y, Reynolds KD, Boyle S, Cappelli C, Cox MG, Dust M, Grenard JL, Mackinnon DP, Stacy AW: Inhibitory control effects in adolescent binge eating and consumption of sugar-sweetened beverages and snacks. Appetite 2014, 81:180-192.

114. Ekelund U, Yngve A, Brage S, Westerterp K, Sjöström M: Body movement and physical activity energy expenditure in children and adolescents: how to adjust for differences in body size and age. Am J Clinical Nutri 2004, 79:851-856.

115. Weyer C, Snitker S, Rising R, Bogardus C, Ravussin E: Determinants of energy expenditure and fuel utilization in man: effects of body composition, age, sex, ethnicity and glucose tolerance in 916 subjects. Int J Obes 1999, 23:715-722.

116. Ravussin E, Bogardus C: Relationship of genetics, age, and physical fitness to daily energy expenditure and fuel utilization. Am J Clin Nutr 1989, 49:968-975.

117. Johansson G, Wikman A, Ahren A-M, Hallmans G, Johansson I: Underreporting of energy intake in repeated 24-hour recalls related to gender, age, weight status, day of interview, educational level, reported food intake, smoking habits and area of living. Public Health Nutr 2001, 4:919-928.

118. Nielsen SJ, Siega-Riz AM, Popkin BM: Trends in energy intake in US between 1977 and 1996: similar shifts seen across age groups. Obes Res 2002, 10:370-378.

119. Benedict C, Brooks SJ, O'Daly OG, Almèn MS, Morell A, Åberg K, Gingnell M, Schultes B, Hallschmid M, Broman J-E: Acute sleep deprivation enhances the brain's response to hedonic food stimuli: an fMRI study. J Clinical Endocrinol Metabol 2012, 97:E443-E447.

120. Malik S, McGlone F, Bedrossian D, Dagher A: Ghrelin modulates brain activity in areas that control appetitive behavior. Cell Metab 2008, 7:400-409.

121. Crabtree DR, Chambers ES, Hardwick RM, Blannin AK: The effects of high-intensity exercise on neural responses to images of food. Am J Clinical Nutri 2014, 99:258-267.

122. Volkow N, Fowler J, Wang G, Baler R, Telang F: Imaging dopamine's role in drug abuse and addiction. Neuropharmacology 2009, 56:3-8.

123. Hollmann M, Hellrung $L$, Pleger $B$, Schlögl H, Kabisch S, Stumvoll M, Villringer A, Horstmann A: Neural correlates of the volitional regulation of the desire for food. Int J Obes 2011, 36:648-655

124. Santel S, Baving L, Krauel K, Münte TF, Rotte M: Hunger and satiety in anorexia nervosa: fMRI during cognitive processing of food pictures. Brain Res 2006, 1114:138-148.

125. Siep N, Roefs A, Roebroeck A, Havermans R, Bonte ML, Jansen A: Hunger is the best spice: an fMRI study of the effects of attention, hunger and calorie content on food reward processing in the amygdala and orbitofrontal cortex. Behav Brain Res 2009, 198:149-158.

126. Killgore WD, Yurgelun-Todd DA: Affect modulates appetite-related brain activity to images of food. Int J Eat Disord 2006, 39:357-363.

doi:10.1186/1475-2891-13-92

Cite this article as: He et al:: Poor ability to resist tempting calorie rich food is linked to altered balance between neural systems involved in urge and self-control. Nutrition Journal 2014 13:92 